\title{
Understanding Flu Vaccination Acceptance Among U.S. Adults: The Health Belief Model and Media Sources
}

\author{
Tong Xie \\ University of Georgia \\ Athens, GA USA \\ Connor Grady \\ University of Georgia \\ Athens, GA USA \\ Michael Cacciatore \\ University of Georgia \\ Athens, GA USA \\ Glen Nowak \\ University of Georgia \\ Athens, GA USA
}

\begin{abstract}
Based on previous studies about the Health Belief Model (HBM) and the reinforcing relationship between media selectivity or preference and individual's behavior, this study used a national representative adult sample to assess flu vaccination as the result of an appraisal of relevant health beliefs, trust towards the authoritative entities (e.g. CDC \& FDA) and vaccine-related media information, in addition to one's existing behavior pattern. Results showed that not-vaccinated individuals differ significantly in their vaccine-related health believes and the trust towards the authoritative information sources. This group acquired less recommendation from health care providers and more negative sentiments about flu vaccine overall. After controlling for the existing behavior, media selectivity and perceived vaccine benefit are predicting individual's vaccination hesitancy and intention in the coming flu season.
\end{abstract}

Keywords - HBM, Media Source, Trust, Flu Vaccine

SUGGESTED CITATION: Xie, T., Grady, C., Cacciatore, M., \& Nowak, G. (2019). Understanding flu vaccination acceptance among U.S. adults: The health belief model and media sources. Proceedings of the International Crisis and Risk Communication Conference, Volume 2 (pp. 35-37). Orlando Fl: Nicholson School of Communication and Media. https://doi.org/10.30658/icrcc.2019.10

\section{INTRODUCTION}

Current flu vaccination acceptance rate among U.S. adult population is considered to be not satisfying according to CDC and Healthy People's report [1,3]. Previous studies have applied the Health Belief Model to understand the predictors of behavior intention to receive an influenza vaccination [5]. Vaccine benefits and recommendations from healthcare professionals are 
crucial factors to be addressed in the health messages [2] and the not-vaccinated group were less confident about the vaccine safety, less likely to believe the necessity of vaccine to fight against flu and more likely to overlook the flu susceptibility [4].

\section{OBJECTIVES}

This study investigated the influenza vaccine/vaccination-related knowledge, belief and recent behaviors of participants. More specifically, we would like to see the association between participants' past vaccination behavior, Health Belief Model measurements (perception of severity, susceptibility, barrier, benefit, self-efficacy and cue to action) and vaccine-related information seeking behavior. The exploration into the information seeking behavior and decision-making process may help current study to propose a theoretical connection between participants' media consumption behavior and participants' existing vaccination behavior.

\section{METHODS}

A national representative sample $(\mathrm{n}=1,005)$ was collected through the National Opinion Research Center's AmeriSpeaks Panel. All the participants are over 19 years old. Descriptive statistics and MANOVA were conducted between the vaccinated and not-vaccinated groups to see if there were some general difference patterns. Overall, influenza vaccine coverage (43.2\%) was consistent with 2016-2017 data from CDC [1]. There were $44.6 \%$ males, and $55.4 \%$ females. $16 . \$ \%$ of the sample participants were $18-29$ years old, $25.3 \% 30-44,27.3 \% 45-59$ and $31.0 \%$ over 60 . The ethnicity consisted of $66.5 \%$ of White participants, $11.2 \%$ of African-Americans, $14.0 \%$ of Hispanics and

$8.3 \%$ of other ethnicity groups. Hierarchical regression models were used to test, after controlling for the current behavior pattern, how would individual's HBM and media preference predict the flu vaccination hesitancy and future vaccination intention.

\section{RESULTS}

Vaccinated participants possessed higher perceived susceptibility ( 2.65 vs 1.86 ), higher perceived flu communicability ( 3.17 vs 2.85), higher perceived severity (3.00 vs 2.59 ) and they faced less of a barrier to vaccination (1.12 vs 1.39), they recognized higher benefit (3.90 vs 2.49 ) and $83.3 \%$ of the vaccinated individuals (vs $37.4 \%$ of not-vaccinated group) had received a recommendation from their health care providers.

In terms of vaccine and overall health-related information seeking behavior and intention, there was also substantial differences between the vaccinated and not-vaccinated groups. Overall the not-vaccinated group used less information from professional healthcare providers and news stories ( 2.53 vs 2.90 and 2.28 vs 2.51 ). They were less likely to use information about vaccines from professional health care providers (3.94 vs 4.24), government agencies (2.60 vs 2.91) and medical or health-related interest sites (3.21 vs 3.58). More not-vaccinated individuals had direct (68.4\% vs $31.6 \%)$ and indirect experience $(66.2 \%$ vs $33.8 \%)$ about bad reactions related to vaccine. Not-vaccinated group received less positive sentiments about flu vaccine overall (3.06 vs 3.55).

The hierarchical regression models showed that individual's existing vaccination behavior $(\beta=.408, p<.000)$ is still the strongest predictor on their vaccination likelihood in the coming flu season. In addition, individual's HBM assessment (i.e. perceived benefit of flu vaccine, $\beta=-.204, \mathrm{p}<.000$ ), trust towards authoritative information sources (i.e. trust towards CDC \& FDA, $\beta=-.212, \mathrm{p}<.000)$ and their existing vaccination behavior $(\beta=-.166, \mathrm{p}<000)$ are some of the strong predictors on individual's overall flu vaccine hesitancy.

\section{DISCUSSION}

This study first showed some considerable patterns that participants who had not received flu vaccine perceived flu vaccine and influenza differently comparing to those who had. Not-vaccinated participants received less vaccine information from health care providers and they had lower trust towards various authoritative entities, such as CDC and FDA. Individual's existing vaccination behavior is still the strongest predictor on their future vaccination likelihood. However, individual's vaccine benefit perception, likelihood to receive more recommendations from health care provider and trust towards the authoritative entities were predicting individual's overall vaccine hesitancy, which in turn leads to lower likelihood to get the vaccine in the future. 


\section{CONCLUSION}

This study found that individuals with different existing flu vaccination behaviors have significantly different believes and perceptions about flu vaccine and influenza. Not-vaccinated individuals have lower level of perceived vaccine benefit, likelihood to get recommendations from health care providers and trust towards the authoritative information sources, which will lead to more vaccine hesitancy and lower intention to vaccinate themselves in the next flu season. Future health campaigns that tries to tackle this public health risk may consider emphasizing more about the perceived benefit of flu vaccine and disseminating the message through local health care providers and pharmacists.

\section{Author Biography}

Tong Xie (mtx73582@uga.edu) is a doctoral student of the Grady College of Journalism and Mass Communication from University of Georgia.

Connor Grady (mconnorgrady@uga.edu) is a student of Microbiology in the Franklin College of Arts and Sciences from University of Georgia.

Michael Cacciatore (mcacciat@uga.edu) is an associate professor of the Grady College of Journalism and Mass Communication from University of Georgia.

Glen Nowak (mgnowak@uga.edu) is a professor of the Grady College of Journalism and Mass Communication from University of Georgia.

\section{REFERENCES}

[1] CDC (2017). FluVaxView: Estimates of flu vaccination coverage among children-United States, 2017-2018 flu season. Available at: https://www.cdc.gov/flu/fluvaxview/coverage-1718estimates-children.htm

[2] Coe, Antoinette B.; Gatewood, Sharon B.S.; Moczygemba, Leticia R.; Goode, Jean-Venable "Kelly" R.. (2012). The use of the health belief model to assess predictors of intent to receive the novel (2009) H1N1 influenza vaccine. University of Minnesota, College of Pharmacy. Retrieved from the University of Minnesota Digital Conservancy. http://hdl.handle.net/11299/128767

[3] Healthy People (2008). Health People 2020: increase the percentage of noninstitutionalized adults aged 18 to 64 years who are vaccinated annually against seasonal influenza. Available at: https://www.healthypeople.gov/2020/topics-objectives/topic/Immunization-and-Infectious-

Diseases/objectives\#4664

[4] Smith, P. J., Humiston, S. G., Marcuse, E. K., Zhao, Z., Dorell, C. G., Howes, C., \& Hibbs, B. (2011). Parental delay or refusal of vaccine doses, childhood vaccination coverage at 24 months of age, and the health belief model. Public Health Reports, 126, 135-146. https://doi.org/10.1177/00333549111260S215

[5] Teitler-Regev, S., Shahrabani, S., \& Benzion, U. (2011). Factors affecting intention among students to be vaccinated against A/H1N1 influenza: a health belief model approach. Advances in Preventive Medicine. https://doi.org/10.4061/2011/353207 